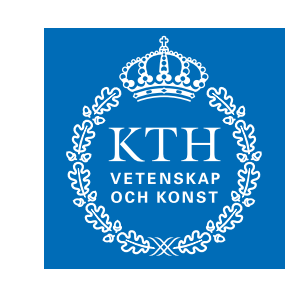

KTH Electrical Engineering

\title{
Linear Precoding in MISO Cognitive Channels with Causal Primary Message
}

(C)2012 IEEE. Personal use of this material is permitted. However, permission to reprint/republish this material for advertising or promotional purposes or for creating new collective works for resale or redistribution to servers or lists, or to reuse any copyrighted component of this work in other works must be obtained from the IEEE.

JING LV, RICARDO BLASCO-SERRANO, EDUARD JORSWIECK, AND RAGNAR THOBABEN

Stockholm 2012

Communication Theory Department

School of Electrical Engineering

KTH Royal Institute of Technology 


\title{
Linear Precoding in MISO Cognitive Channels with Causal Primary Message
}

\author{
Jing Lv*, Ricardo Blasco-Serrano ${ }^{\dagger}$, Eduard Jorswieck*, and Ragnar Thobaben ${ }^{\dagger}$ \\ ${ }^{*}$ Communications Theory, Communications Laboratory, Dresden University of Technology, Dresden, Germany \\ E-mail: \{jing.lv, eduard.jorswieck\}@tu-dresden.de \\ ${ }^{\dagger}$ School of Electrical Engineering and ACCESS Linnaeus Centre, KTH Royal Institute of Technology, Stockholm, Sweden \\ E-mail: \{ricardo.blasco, ragnar.thobaben\}@ee.kth.se
}

\begin{abstract}
The coexistence of a single-input single-output (SISO) primary link and a multiple-input single-output (MISO) secondary link is considered in an extended cognitive radio channel setup, where the secondary transmitter has to obtain ("learn") the primary message in a first phase rather than having non-causal knowledge of it. An achievable rate region is derived that combines decodeand-forward relaying with linear precoding in the second phase. The optimal transmission strategy is found that maximizes the secondary rate with the primary rate requirement. The performance of the proposed strategy is compared with that in [1], where dirtypaper coding (DPC) is deployed in the second phase, in terms of average secondary rate. The performance degradation is negligible at certain SNR and primary link load, and the implementation is of lower complexity. The comparison with the underlay strategy in [2] is also performed, where the secondary transmitter has no knowledge of the primary message.
\end{abstract}

\section{INTRODUCTION}

Cognitive radio ( $\mathrm{CR}$ ) is brought up to enable the coexistence of primary and secondary users through spectrum sharing, thus increasing the spectral efficiency. We consider the scenarios of underlay and overlay CR, where the difference lies in whether the secondary transmitter has knowledge of the primary message. The tolerable quality of service (QoS) degradation of the primary users is characterized by interference temperature constraints (ITC) [3, 4]. A proper information theoretical model for underlay $\mathrm{CR}$ is the interference channel (IC), where independent transceiver pairs interfere with each other $[5,6]$. There are also studies on linear transceiver design for underlay CR. By deploying multiple antennas at the secondary transmitter, beamforming technique was used to control the interference to the primary receivers while maximizing the secondary rate $[7,8]$. For the special case of a multiple-input single-output (MISO) secondary link coexisting with a single-input single-output (SISO) primary link, a closed-form solution was derived for the optimal beamforming at the secondary transmitter. Furthermore, additional gains could be achieved if rate splitting and successive decoding is viable in the secondary link [2].

In contrast, the cognitive radio channel (also known as interference channel with degraded message sets (IC-DMS)) is used to model overlay $\mathrm{CR}$, assuming that the secondary transmitter has non-causal knowledge of the primary message. For the two

Part of this work has been performed in the framework of Network of Excellence ACROPOLIS, which is partly funded by the European Union under its FP7 ICT Objective 1.1 - The Network of the Future. user SISO IC-DMS, in the low interference regime, it was shown in $[9,10]$ that the optimal strategy at the secondary transmitter consists of combining selfless relaying of the primary message and interference pre-cancellation using dirty paper coding (DPC) [11] to transmit its own message. In contrast, the capacity of part of the strong interference regime was obtained in [12] using superposition coding and interference decoding. In [13], an achievable rate region was characterized for the coexistence of a SISO primary link and a MISO secondary link, by combining selfless relaying of the primary message with DPC to protect the secondary transmission from the interference due to the primary message, and optimal beamforming and power allocation was derived to maximize the secondary rate while satisfying the primary rate requirement. Due to the complexity of DPC, linear precoding at the secondary transmitter was studied in [14], where the rate loss becomes almost constant at high signal-to-noiseratios (SNR).

A comparison of the transmission strategies for overlay and underlay CR suggests that non-causal knowledge of the primary message at the secondary transmitter leads to a significant gain in the achievable secondary rate [13]. However, a critical point is how the secondary transmitter obtains the primary message. Learning the primary message requires the secondary transmitter to be passive for certain time, and it leads therefore inevitably to a rate loss for the secondary link which may lower the attractiveness of the overlay strategy. Unfortunately, fundamental studies of overlay CR do not account for this rate loss, and the best known achievable rates may therefore be too optimistic. Motivated by this observation, an extension of the cognitive radio channel model was considered in [1] which takes into account the initial transmission phase for exchanging the primary message as well. For this extended cognitive radio channel model, the achievable transmission scheme was studied that combines decode-and-forward (DF) relaying $[15,16]$ with the overlay strategy in [13]. Achievable rates derived for the proposed strategy were then used to compare the performance of the underlay and overlay strategies, using a simple geometrical model for the relative positions of the nodes.

The main contribution of this work is the study of the impact when linear precoding is deployed at the secondary transmitter instead of DPC for the system model considered in [1], given the high complexity of practical implementation of DPC. The optimal transmission strategy is derived to maximize the sec- 
ondary rate while satisfying the primary rate requirement. The performance of the proposed strategy is compared with that of the overlay strategy in [1] and the underlay strategy in [2], in terms of average secondary rate, through numerical evaluation.

\section{PRELIMINARIES}

\section{A. Notation}

Vectors and matrices are represented in lower and upper case boldface letters, respectively. $|\cdot|$ is the absolute value of a scalar, $\|\cdot\|$ is the Frobenius norm of a vector or matrix, and $(\cdot)^{H}$ stands for Hermitian transpose. $\Pi_{\boldsymbol{a}} \triangleq \boldsymbol{a}\left(\boldsymbol{a}^{H} \boldsymbol{a}\right)^{-1} \boldsymbol{a}^{H}$ denotes the orthogonal projection onto the space of $a$, and $\Pi_{a}^{\perp} \triangleq I-\Pi_{a}$, where $I$ is the identity matrix, denotes the orthogonal projection onto the orthogonal complement of the space of $\boldsymbol{a}$. In this paper all logarithms are taken to the base of 2 and all rates are expressed in bits.

\section{B. System Model}

We consider the coexistence of a SISO primary link and a MISO secondary link in the scenario of overlay CR. As depicted in Figure 1, the transmission is divided into two phases. The first phase is to inform the secondary transmitter of the primary message $d_{1}$, and the second phase corresponds to the cognitive radio channel setup, where linear precoding is deployed at the secondary transmitter for the transmission of $d_{1}$ and the secondary message $d_{2}$. The secondary transmitter has $N$ antennas and is half duplex. The received signal in the first phase is

$$
\begin{aligned}
y_{1}^{(1)} & =h_{11} x_{1}^{(1)}+n_{1} \\
\boldsymbol{y}_{s t} & =\boldsymbol{h}_{t t} x_{1}^{(1)}+\boldsymbol{n}_{s t}
\end{aligned}
$$

where $x_{1}^{(1)}$ is the signal transmitted by the primary transmitter, $n_{1}$ and $\boldsymbol{n}_{s t}$ are the noises, and $y_{1}^{(1)}$ and $\boldsymbol{y}_{s t}$ are the received signals at the primary receiver and secondary transmitter, respectively. Similarly, for the second phase

$$
\begin{aligned}
& y_{1}^{(2)}=h_{11} x_{1}^{(2)}+\boldsymbol{h}_{21}^{H} \boldsymbol{x}_{2}^{(2)}+n_{1} \\
& y_{2}^{(2)}=h_{12} x_{1}^{(2)}+\boldsymbol{h}_{22}^{H} \boldsymbol{x}_{2}^{(2)}+n_{2}
\end{aligned}
$$

where $x_{1}^{(2)}$ and $\boldsymbol{x}_{2}^{(2)}$ are the signals transmitted by the primary and secondary transmitters, and $y_{1}^{(2)}$ and $y_{2}^{(2)}$ are the received signals at the respective receivers.

Phase 1:

Phase 2:

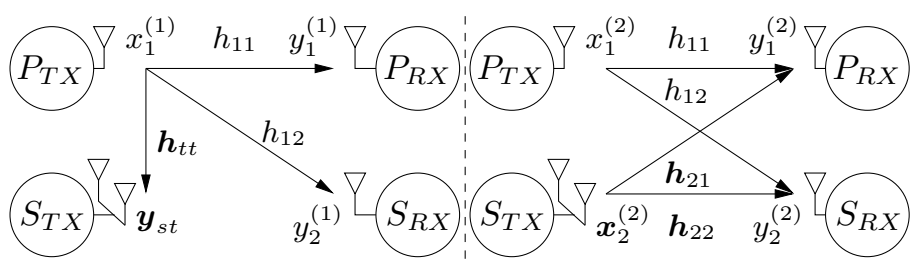

Fig. 1. Illustration of the two-phase transmission scheme.

The noises are independent complex white Gaussian with unit variance: where $n_{1}, n_{2} \sim \mathcal{C N}(0,1)$ and $\boldsymbol{n}_{s t} \sim \mathcal{C N}(\mathbf{0}, \boldsymbol{I})$. $h_{i j}\left(\boldsymbol{h}_{i j}\right.$ for vector channel) denotes the channel gain from transmitter $i \in\{1,2\}$ to receiver $j \in\{1,2\}$, which is assumed to be constant during the two phases, and the vector channel between the two transmitters in the first phase is denoted as $\boldsymbol{h}_{t t}$, all of which are complex Gaussian distributed: $h_{11} \sim \mathcal{C N}(0,1)$, $h_{12} \sim \mathcal{C N}(0,1), h_{21} \sim \mathcal{C N}(\mathbf{0}, \boldsymbol{I}), \boldsymbol{h}_{22} \sim \mathcal{C N}(\mathbf{0}, \boldsymbol{I})$ and $\boldsymbol{h}_{t t} \sim \mathcal{C} \mathcal{N}(\mathbf{0}, \boldsymbol{I})$.

The entire transmission is carried out over $n$ channel uses, $k$ channel uses are used during the first phase, and $(n-k)$ channel uses are used during the second phase. The fraction of channel uses in the first and second phases are given by $\alpha=k / n$ and $1-\alpha$, respectively.

\section{Transmission Strategy And Problem FORMULATION}

\section{A. Transmission Strategy}

The transmission strategy combines DF relaying $[15,16]$ with the overlay strategy in [14] for the extended cognitive radio channel model.

In the first phase, which has relative duration $\alpha$, the primary transmitter uses a code $\mathcal{C}_{0}$ with rate $R_{0}=R_{1}^{\star} / \alpha$ and power $P_{1}^{(1)}$ to broadcast $d_{1}$ to both the primary receiver and the secondary transmitter, i.e. $x_{1}^{(1)}=\sqrt{P_{1}^{(1)}} u_{11}\left(d_{1}\right)$, where $R_{1}^{\star}$ is the primary rate requirement. Define the rates

$$
\begin{aligned}
R_{1}^{(1)} & =\alpha \log \left(1+P_{1}^{(1)}\left|h_{11}\right|^{2}\right), \\
R_{t} & =\alpha \log \left(1+P_{1}^{(1)}\left\|\boldsymbol{h}_{t t}\right\|^{2}\right),
\end{aligned}
$$

that characterize the primary transmission rate and the rate between the two transmitters in the first phase, respectively, assuming maximum ratio combining is used at the secondary transmitter for reception. To ensure decodability at the secondary transmitter after the first phase, $R_{t} \geq R_{1}^{\star}$ has to be satisfied. Assuming $\| \boldsymbol{h}_{t t}||>\left|h_{11}\right|$, after the first phase, the primary receiver only has partial knowledge (i.e. its observation) about $d_{1}$. In the second phase, the primary and secondary transmitters cooperate to resolve this ambiguity at the primary receiver. The code $\mathcal{C}_{0}$ is subdivided into disjoint (lower-rate) sub-codes $\mathcal{C}_{i}$ (bins), which are designed to be good channel codes. That is, each codeword of $\mathcal{C}_{0}$ belongs to a sub-code $\mathcal{C}_{i}$. The index $i$ of the sub-code containing the codeword sent in the first phase is transmitted cooperatively by the primary transmitter (using power $P_{1}^{(2)}$ ) and the secondary transmitter (using a fraction $p_{a}$ of its available power $P_{2}$ ). Knowing this index, the primary receiver can decode $d_{1}$ based on its channel observation from the first phase, by considering only codewords from the (low-rate) sub-code $\mathcal{C}_{i}$. In addition, the secondary transmitter employs the remaining power $p_{b}$ for the transmission of $d_{2}$. Relayed and private messages are broadcasted by the secondary transmitter using independent beamformers $\boldsymbol{w}_{1}$ and $\boldsymbol{w}_{2}$, i.e. $x_{1}^{(2)}=\sqrt{P_{1}^{(2)}} u_{12}\left(d_{1}\right), \boldsymbol{x}_{2}^{(2)}=\sqrt{p_{a}} \boldsymbol{w}_{1} u_{12}\left(d_{1}\right)+\sqrt{p_{b}} \boldsymbol{w}_{2} u_{2}\left(d_{2}\right)$. 
Define the rates

$$
\begin{aligned}
& R_{1}^{(2)}=(1-\alpha) \log \left(1+\frac{\left|\sqrt{P_{1}^{(2)}} h_{11}+\sqrt{\frac{p_{a}}{1-\alpha}} \boldsymbol{h}_{21}^{H} \boldsymbol{w}_{1}\right|^{2}}{1+\frac{p_{b}}{1-\alpha}\left|\boldsymbol{h}_{21}^{H} \boldsymbol{w}_{2}\right|^{2}}\right) \\
& R_{2}=(1-\alpha) \log \left(1+\frac{\frac{p_{b}}{1-\alpha}\left|\boldsymbol{h}_{22}^{H} \boldsymbol{w}_{2}\right|^{2}}{1+\left|\sqrt{P_{1}^{(2)}} h_{12}+\sqrt{\frac{p_{a}}{1-\alpha}} h_{22}^{H} \boldsymbol{w}_{1}\right|^{2}}\right),
\end{aligned}
$$

that characterize the cooperative transmission rate (i.e. transmission of the bin index) and the secondary rate achievable in the second phase by our strategy, respectively. Note that the powers are scaled up to take into account the duration of the second phase. Using DF relaying arguments $[15,16]$, it is possible to show that the achievable primary rate is

$$
R_{1}=R_{1}^{(1)}+R_{1}^{(2)} .
$$

The achievable rate region is $\mathcal{R}=\left(R_{1}, R_{2}\right)$, for any feasible choice of beamformers, phase splitting, and power allocation that ensures decodability of the primary message at the secondary transmitter after the first phase.

\section{B. Problem Formulation}

The problem of maximizing the secondary rate $R_{2}$ while satisfying the primary rate requirement $R_{1}^{\star}$ is formulated as

$$
\begin{array}{ll}
\max _{\alpha} R_{2} \\
\boldsymbol{w}_{1}, p_{a} \\
\boldsymbol{w}_{2}, p_{b} \\
P_{1}^{(1)}, P_{1}^{(2)} \\
\text { s.t. } R_{t} \geq R_{1}^{\star}, \\
\quad R_{1} \geq R_{1}^{\star}, \\
\quad\left\|\boldsymbol{w}_{1}\right\|=\left\|\boldsymbol{w}_{2}\right\|=1, \\
\quad \alpha P_{1}^{(1)}+(1-\alpha) P_{1}^{(2)} \leq P_{1}, P_{1}^{(1)}>0, P_{1}^{(2)} \geq 0, \\
\quad p_{a}+p_{b} \leq P_{2}, p_{a} \geq 0, p_{b} \geq 0, \\
\quad 0<\alpha<1,
\end{array}
$$

where (10b) ensures the decodability of the primary message at the secondary transmitter after the first phase, (10c) fulfils the primary rate requirement, and (10e) and (10f) are the power constraints at the primary and secondary transmitters, respectively.

\section{System OPTIMIZATION}

In this section we solve the problem formulated in Section III-B. First we introduce the following lemma.

\section{Lemma 1. The function}

$$
\beta \log \left(1+\frac{P}{\beta}\right)
$$

defined for $\beta \in(0,1]$ and $P>0$, is strictly increasing in $\beta$.

Using this lemma, we prove the following proposition.
Proposition 1. If the channels $\boldsymbol{h}_{21}$ and $\boldsymbol{h}_{22}$ are not colinear, both the primary and secondary transmitters use full power at the optimum, i.e.

1) $\alpha P_{1}^{(1)}+(1-\alpha) P_{1}^{(2)}=P_{1}$,

2) $p_{a}+p_{b}=P_{2}$.

Proof: First we prove Statement 1. Let the set of parameters $\left(\alpha, P_{1}^{(1)}, P_{1}^{(2)}, p_{a}, p_{b}, \boldsymbol{w}_{1}, \boldsymbol{w}_{2}\right)$ attain the optimum and be such that $\alpha P_{1}^{(1)}+(1-\alpha) P_{1}^{(2)}<P_{1}$.

The proof is divided into several cases.

a) $P_{1}^{(2)}>0$ : In this case we can find $\tilde{P}_{1}^{(1)}>P_{1}^{(1)}$ such that the corresponding rate $\tilde{R}_{1}^{(1)}$ in the first phase satisfies $\tilde{R}_{1}^{(1)}+R_{1}^{(2)}>R_{1}^{\star}$.

Now let $\tilde{P}_{1}^{(2)}=\gamma P_{1}^{(2)}$ and $\tilde{p}_{a}=\gamma p_{a}$. Let $\tilde{R}_{1}^{(2)}$ denote the secondary rate evaluated for $\tilde{P}_{1}^{(2)}$ and $\tilde{p}_{a}$. Since $R_{1}^{(2)}$ is continuous on both $P_{1}^{(2)}$ and $p_{a}$, we can find $0 \leq \gamma<1$ such that $\tilde{R}_{1}^{(1)}+\tilde{R}_{1}^{(2)}>R_{1}^{\star}$. At the same time, the new rate $\tilde{R}_{2}$ for the secondary link is

$$
\tilde{R}_{2}=(1-\alpha) \log \left(1+\frac{\frac{p_{b}}{1-\alpha}\left|\boldsymbol{h}_{22}^{H} \boldsymbol{w}_{2}\right|^{2}}{1+\gamma\left|\sqrt{P_{1}^{(2)}} h_{12}+\sqrt{\frac{p_{a}}{1-\alpha}} \boldsymbol{h}_{22}^{H} \boldsymbol{w}_{1}\right|^{2}}\right) .
$$

We have $\tilde{R}_{2}>R_{2}$ assuming that either $h_{12} \neq 0$ and/or $\boldsymbol{h}_{22}^{H} \boldsymbol{w}_{1} \neq$ 0 , and if $h_{12}=\boldsymbol{h}_{22}^{H} \boldsymbol{w}_{1}=0$, we can find a shorter duration for the first phase that satisfies (10b)-(10g). From Lemma 1 we know that this yields a larger secondary rate.

In either case, we have contradicted our initial assumption on the optimality of the set of parameters.

b) $P_{1}^{(2)}=0$ and $p_{a}>0$ : In this case we can find $\tilde{P}_{1}^{(1)}>$ $P_{1}^{(1)}$ such that the corresponding rate $\tilde{R}_{1}^{(1)}$ in the first phase again satisfies $\tilde{R}_{1}^{(1)}+R_{1}^{(2)}>R_{1}^{\star}$.

Now we can find $\tilde{p}_{a}<p_{a}$ such that the corresponding rate $\tilde{R}_{1}^{(2)}$ in the second phase satisfies $\tilde{R}_{1}^{(1)}+\tilde{R}_{1}^{(2)}>R_{1}^{\star}$. However, assuming $\boldsymbol{h}_{22}^{H} \boldsymbol{w}_{1} \neq 0$, this leads to a secondary rate $\tilde{R}_{2}$ that is larger than the one that would be obtained for $p_{a}$. Again we have reached a contradiction. If $\boldsymbol{h}_{22}^{H} \boldsymbol{w}_{1}=0$, we can proceed as in the previous case, shortening the first phase and invoking Lemma 1 to reach a similar contradiction.

c) $P_{1}^{(2)}=p_{a}=0$ : Note that in this case $R_{1}^{(2)}=0$ and therefore $R_{1}^{(1)} \geq R_{1}^{\star}$. We can find $\tilde{P}_{1}^{(1)}>P_{1}^{(1)}$ such that the corresponding rates $\tilde{R}_{1}^{(1)}$ and $\tilde{R}_{t}$ in the first phase satisfy $\tilde{R}_{1}^{(1)}>R_{1}^{\star}$ and $\tilde{R}_{t}>R_{1}^{\star}$.

Now we can find $\tilde{\alpha}<\alpha$ such that the new rates $\hat{R}_{1}^{(1)}$ and $\hat{R}_{t}$ in the first phase still satisfy $\hat{R}_{1}^{(1)} \geq R_{1}^{\star}$ and $\hat{R}_{t} \geq R_{1}^{\star}$. However, for $P_{1}^{(2)}=p_{a}=0$, we know from Lemma 1 that $R_{2}$ increases with decreasing $\alpha$. Once more, this contradicts our initial statement.

Now we prove Statement 2. Again, let the set of parameters $\left(\alpha, P_{1}^{(1)}, P_{1}^{(2)}, p_{a}, p_{b}, \boldsymbol{w}_{1}, \boldsymbol{w}_{2}\right)$ attain the optimum and be such that $p_{a}+p_{b}<P_{2}$. Then we can increase the power $p_{b}$ in a direction orthogonal to $\boldsymbol{h}_{21}$. In this way we increase the rate $R_{2}$ without lowering $R_{1}^{(2)}$ (hence without violating contraint (10c)). This contradicts our assumption on the optimality of the set of parameters. 
Remark 1. If the channels $\boldsymbol{h}_{21}$ and $\boldsymbol{h}_{22}$ are colinear, we lose the advantage of having multiple transmit antennas, since we cannot increase the secondary rate without creating interference. In this case it is possible to find cases where the optimal parameters satisfy $p_{a}+p_{b}<P_{2}$.

Now we introduce the following proposition that solves problem (10).

Proposition 2. Assuming that the channels $\boldsymbol{h}_{21}$ and $\boldsymbol{h}_{22}$ are not colinear, for certain $\alpha \in(0,1)$ and $P_{1}^{(1)} \in\left(0, \frac{P_{1}}{\alpha}\right]$,

1) if $R_{1}^{(1)} \geq R_{1}^{\star}$,

$$
\boldsymbol{w}_{2}=\frac{\boldsymbol{h}_{22}}{\left\|\boldsymbol{h}_{22}\right\|}, p_{b}=P_{2}
$$

2) if $R_{1}^{(1)}<R_{1}^{\star}$ and $R_{t} \geq R_{1}^{\star}$, (10) is solved by

$$
\begin{aligned}
\boldsymbol{w}_{1}\left(\psi_{1}, \psi_{2}, \lambda_{1}\right)= & e^{j \psi_{1}} \sqrt{\lambda_{1}} \frac{\Pi_{\boldsymbol{h}_{22}}^{\perp} \boldsymbol{h}_{21}}{\left\|\Pi_{\boldsymbol{h}_{22}}^{\perp} \boldsymbol{h}_{21}\right\|} \\
& +e^{j \psi_{2}} \sqrt{1-\lambda_{1}} \frac{\Pi_{\boldsymbol{h}_{22}} \boldsymbol{h}_{21}}{\left\|\Pi_{\boldsymbol{h}_{22}} \boldsymbol{h}_{21}\right\|},
\end{aligned}
$$

where

$$
\psi_{1}=\frac{\varphi}{|\varphi|}
$$

$\varphi=\sqrt{P_{1}^{(2)}} h_{11}+\sqrt{\frac{p_{a}}{1-\alpha}} e^{j \psi_{2}} \sqrt{1-\lambda_{1}} \boldsymbol{h}_{21}^{H} \frac{\Pi_{\boldsymbol{h}_{22}} \boldsymbol{h}_{21}}{\left\|\Pi_{\boldsymbol{h}_{22}} \boldsymbol{h}_{21}\right\|}$,

and by

$$
\begin{gathered}
\boldsymbol{w}_{2}\left(\lambda_{2}\right)=\sqrt{\lambda_{2}} \frac{\Pi_{\boldsymbol{h}_{21}} \boldsymbol{h}_{22}}{\left\|\Pi_{\boldsymbol{h}_{21}} \boldsymbol{h}_{22}\right\|}+\sqrt{1-\lambda_{2}} \frac{\Pi_{\boldsymbol{h}_{21}}^{\perp} \boldsymbol{h}_{22}}{\left\|\Pi_{\boldsymbol{h}_{21}}^{\perp} \boldsymbol{h}_{22}\right\|}, \\
p_{b}=P_{2}-p_{a}
\end{gathered}
$$

where

$$
\begin{aligned}
& \lambda_{2}= \begin{cases}\lambda_{M R T} & \text { if } \lambda_{M R T} \leq \frac{z(1-\alpha)}{\left\|\boldsymbol{h}_{21}\right\|^{2} p_{b}} \\
\frac{z(1-\alpha)}{\left\|\boldsymbol{h}_{21}\right\|^{2} p_{b}} & \text { otherwise }\end{cases} \\
& \lambda_{M R T}=\frac{\left\|\Pi_{\boldsymbol{h}_{21}} \boldsymbol{h}_{22}\right\|^{2}}{\left\|\boldsymbol{h}_{22}\right\|^{2}}, \\
& z=\frac{\left|\sqrt{P_{1}^{(2)}} h_{11}+\sqrt{\frac{p_{a}}{1-\alpha}} \boldsymbol{h}_{21}^{H} \boldsymbol{w}_{1}\left(\psi_{1}, \psi_{2}, \lambda_{1}\right)\right|^{2}}{2^{\frac{R_{1}^{\star}-R_{1}^{(1)}}{1-\alpha}}-1}-1 \geq 0, \\
& P_{1}^{(2)}=\frac{P_{1}-\alpha P_{1}^{(1)}}{1-\alpha},
\end{aligned}
$$

for certain $p_{a} \in\left[0, P_{2}\right], \psi_{2} \in[0,2 \pi]$, and $\lambda_{1} \in[0,1]$.

The optimal objective value in (10a) can be obtained by searching over $\alpha, P_{1}^{(1)}, p_{a}, \psi_{2}$, and $\lambda_{1}$.

Proof: Case 1 is straightforward. For Case 2, given $\alpha$ and $P_{1}^{(1)}, P_{1}^{(2)}$ is known by Proposition 1 , and the problem has the similar form as (4) in [14], which can be solved by Proposition 1 in [14].

Remark 2. For Case 2 in Proposition 2, a search over 5 parameters is needed to obtain the optimal objective value, which is quite computation-prohibitive. By semidefinite relaxation, problem (4) in [14] is reformulated as a semidefinite programming (SDP) problem, which can be solved by CVX efficiently [17], and then the optimal rank-one solution can be obtained by rank-one decomposition [18], when the number of antennas at the secondary transmitter is more than two. Since the problem in Case 2 has the similar form as (4) in [14] given $\alpha$ and $P_{1}^{(1)}$, it can be solved by semidefinite relaxation and rank-one decomposition, for certain $\alpha \in(0,1)$ and $P_{1}^{(1)} \in\left(0, \frac{P_{1}}{\alpha}\right]$, with more than two antennas at the secondary transmitter; moreover, the noncolinearity of the channels $\boldsymbol{h}_{21}$ and $\boldsymbol{h}_{22}$ is not required, as long as the power constraint (10f) is fulfiled. This means a search over 2 parameters is needed, where a SDP problem is solved for each set of parameters; then the optimal beamforming vectors can be obtained by applying rank-one decomposition to the resulted SDP solution at the optimal objective value.

\section{Numerical EVALUATION}

We first compare the performance of the overlay strategy proposed in Section III-A, with that of the overlay strategy in [1] where DPC is deployed at the secondary transmitter to protect the secondary transmission from the interference due to the primary message, in terms of average secondary rate. The two strategies are labeled as "LP overlay" and "DPC overlay", respectively. For the simulation, we set $N=3$ and $P_{1}=10 \mathrm{~dB}$, and vary $P_{2}$ as the SNR of the secondary link. The primary link load is defined as the ratio of the primary rate requirement and the actual channel capacity of the primary link for the instantaneous channel realization. As shown in Figure 2, the performance degradation of the proposed strategy is negligible at certain SNR and primary link load, and the implementation is of lower complexity compared to the overlay strategy based on DPC.

We now compare the performance of the proposed strategy with that of the underlay strategy in [2], where the secondary transmitter has no knowledge of the primary message, and rate splitting and successive decoding is deployed in the secondary link whenever viable. As shown in Figure 3, average secondary rate, as well as the percentage when the performance of the proposed strategy is better than the underlay strategy, is shown at certain SNR and primary link load.

\section{CONCLUSION}

In this work we have considered the coexistence of a SISO primary link and a MISO secondary link in the extended cognitive radio channel setup, where the transmission is divided into two phases. In the first phase, the secondary transmitter learns the primary message by listening to the primary transmission; in the second phase, the secondary transmitter and the primary transmitter cooperate to complete the primary transmission and the secondary transmission. We have found the set of phase splitting, power allocation, and beamformers that maximizes the secondary rate while satisfying a primary rate requirement. We have compared the performance of the proposed strategy with an overlay strategy based on DPC, where the performance degradation of the proposed strategy is negligible at certain 


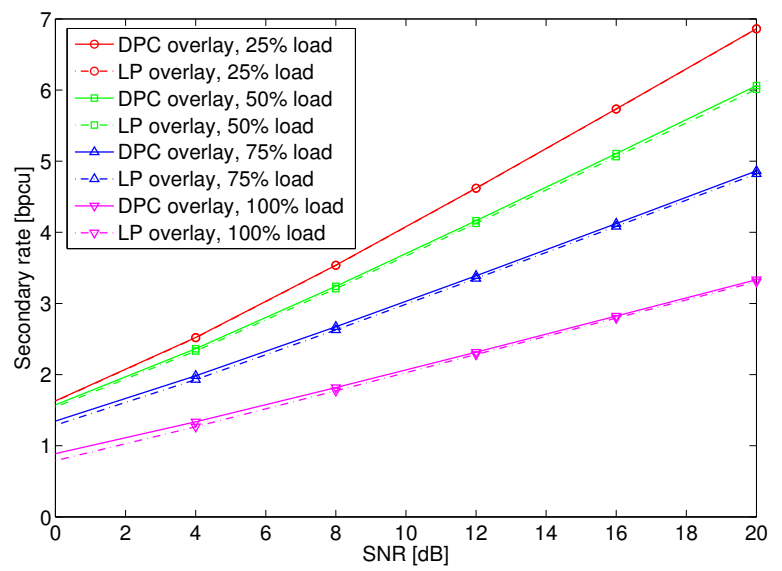

Fig. 2. Average secondary rate versus SNR of the secondary link.
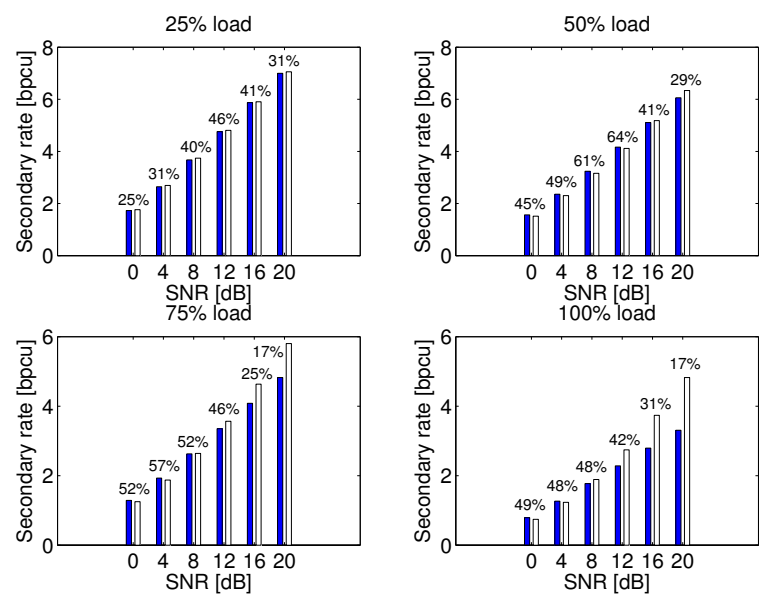

Fig. 3. Average secondary rate versus SNR of the secondary link: the blue bars represent the proposed strategy, and the white bars represent the underlay strategy.

SNR and primary link load, and the implementation is of lower complexity, as well as with an underlay strategy where the secondary transmitter has no knowledge of the primary message.

\section{REFERENCES}

[1] R. Blasco-Serrano, J. Lv, R. Thobaben, E. Jorswieck, A. Kliks, and M. Skoglund, "Comparison of underlay and overlay spectrum sharing strategies in MISO cognitive channels," in Proc. of International Conference on Cognitive Radio Oriented Wireless Networks (CROWNCOM), June 2012.

[2] J. Lv and E. Jorswieck, "Spatial shaping in cognitive system with coded legacy transmission," in Proc. of International ITG Workshop on Smart Antennas (WSA), Feb. 2011.

[3] S. Haykin, "Cognitive radio: brain-empowered wireless communications," IEEE J. Sel. Areas in Commun., vol. 23, no. 2, pp. 201-220, Feb. 2005.
[4] A. Goldsmith, S. A. Jafar, I. Maric, and S. Srinivasa, "Breaking spectrum gridlock with cognitive radios: An information theoretic perspective," Proc. IEEE, vol. 97, no. 5, pp. 894-914, May 2009.

[5] A. Carleial, "Interference channels," IEEE Transactions on Information Theory, vol. 24, no. 1, pp. 60-70, Jan. 1978.

[6] T. S. Han and K. Kobayashi, "A new achievable rate region for the interference channel," IEEE Transactions on Information Theory, vol. 27, no. 1, pp. 49-60, Jan. 1981.

[7] R. Zhang and Y.-C. Liang, "Exploiting multi-antennas for opportunistic spectrum sharing in cognitive radio networks," IEEE Journal on Selected Topics in Signal Processing, vol. 2, no. 1, pp. 88-102, Feb. 2008.

[8] R. Zhang, Y.-C. Liang, and S. Cui, "Dynamic resource allocation in cognitive radio networks," IEEE Signal Processing Magazine, vol. 27, no. 3, pp. 102-114, May 2010.

[9] W. Wu, S. Vishwanath, and A. Arapostathis, "Capacity of a class of cognitive radio channels: Interference channels with degraded message sets," IEEE Transactions on Information Theory, vol. 53, no. 11, pp. 4391-4399, Nov. 2007.

[10] A. Jovicic and P. Viswanath, "Cognitive radio: An information-theoretic perspective," IEEE Transactions on Information Theory, vol. 55, no. 9, pp. 3945-3958, Sept. 2009.

[11] M. Costa, "Writing on dirty paper (corresp.)," IEEE Transactions on Information Theory, vol. 29, no. 3, pp. 439-441, May 1983.

[12] I. Maric, R. D. Yates, and G. Kramer, "Capacity of interference channels with partial transmitter cooperation," IEEE Transactions on Information Theory, vol. 53, no. 10, pp. 3536-3548, Oct. 2007.

[13] J. Lv, R. Blasco-Serrano, E. Jorswieck, R. Thobaben, and A. Kliks, "Optimal beamforming in MISO cognitive channels with degraded message sets," in Proc. IEEE Wireless Communications and Networking Conference (WCNC), Apr. 2012.

[14] J. Lv, E. Jorswieck, R. Blasco-Serrano, R. Thobaben, and A. Kliks, "Linear precoding in MISO cognitive channels with degraded message sets," in Proc. of International ITG Workshop on Smart Antennas (WSA), Mar. 2012.

[15] T. Cover and A. El Gamal, "Capacity theorems for the relay channel," IEEE Transactions on Information Theory, vol. 25, no. 5, pp. 572-584, Sept. 1979.

[16] A. Høst-Madsen and J. Zhang, "Capacity bounds and power allocation for wireless relay channels," IEEE Transactions on Information Theory, vol. 51, no. 6, pp. 2020-2040, June 2005.

[17] M. Grant and S. Boyd, "CVX: Matlab software for disciplined convex programming, version 1.22," http://cvxr.com/cvx, Feb. 2012.

[18] W. Ai, Y. Huang, and S. Zhang, "New results on Hermitian matrix rank-one decomposition," Mathematical Programming, vol. 128, pp. 253-283, 2011. 